\title{
Article \\ Identification of Risk Factors Associated with Resistant Escherichia coli Isolates from Poultry Farms in the East Coast of Peninsular Malaysia: A Cross Sectional Study
}

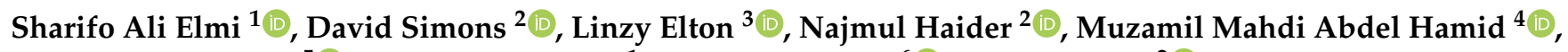 \\ Yassir Adam Shuaib ${ }^{5}$ D, Mohd Azam Khan ${ }^{1}$, Iekhsan Othman ${ }^{6}{ }^{\mathbb{D}}$, Richard Kock ${ }^{2}{ }^{\mathbb{D}}$ and \\ Abdinasir Yusuf Osman 2,*(i) \\ 1 Faculty of Veterinary Medicine, Universiti Malaysia Kelantan, Pengkalan Chepa, Kota Bharu 16100, Kelantan, \\ Malaysia; sharifo.d18d006f@siswa.umk.edu.my (S.A.E.); azamkhan@umk.edu.my (M.A.K.) \\ 2 The Royal Veterinary College, University of London, Hawkshead Lane, North Mymms, Hatfield AL9 7TA, \\ Hertfordshire, UK; dsimons19@rvc.ac.uk (D.S.); nhaider@rvc.ac.uk (N.H.); rkock@rvc.ac.uk (R.K.) \\ 3 Centre for Clinical Microbiology, University College London, London NW3 2PF, UK; linzy.elton@ucl.ac.uk \\ 4 Institute of Endemic Diseases, University of Khartoum, Khartoum 11111, Sudan; mahdi@iend.org \\ 5 College of Veterinary Medicine, Sudan University of Science and Technology, Hilat Kuku, \\ Khartoum North 13321, Sudan; vet.aboamar@gmail.com \\ 6 Jeffrey Cheah School of Medicine and Health Sciences, Monash University, Selangor 47500, Malaysia; \\ iekhsan.othman@monash.edu \\ * Correspondence: aosman@rvc.ac.uk; Tel.: +44-742-404-9130
}

check for updates

Citation: Elmi, S.A.; Simons, D.; Elton, L.; Haider, N.; Abdel Hamid, M.M.; Shuaib, Y.A.; Khan, M.A.; Othman, I.; Kock, R.; Osman, A.Y. Identification of Risk Factors Associated with Resistant Escherichia coli Isolates from Poultry Farms in the East Coast of Peninsular Malaysia: A Cross Sectional Study. Antibiotics 2021, 10, 117. https://doi.org/ 10.3390/antibiotics10020117

Received: 26 December 2020 Accepted: 21 January 2021 Published: 26 January 2021

Publisher's Note: MDPI stays neutral with regard to jurisdictional claims in published maps and institutional affiliations.

Copyright: (c) 2021 by the authors. Licensee MDPI, Basel, Switzerland. This article is an open access article distributed under the terms and conditions of the Creative Commons Attribution (CC BY) license (https:/ / creativecommons.org/licenses/by/ $4.0 /)$.

\begin{abstract}
Antimicrobial resistance is of concern to global health security worldwide. We aimed to identify the prevalence, resistance patterns, and risk factors associated with Escherichia coli (E. coli) resistance from poultry farms in Kelantan, Terengganu, and Pahang states of east coast peninsular Malaysia. Between 8 February 2019 and 23 February 2020, a total of 371 samples (cloacal swabs = 259; faecal $=84$; Sewage $=14$, Tap water $=14$ ) were collected. Characteristics of the sampled farms including management type, biosecurity, and history of disease were obtained using semistructured questionnaire. Presumptive E. coli isolates were identified based on colony morphology with subsequent biochemical and PCR confirmation. Susceptibility of isolates was tested against a panel of 12 antimicrobials and interpreted alongside risk factor data obtained from the surveys. We isolated 717 E. coli samples from poultry and environmental samples. Our findings revealed that cloacal $(17.8 \%, 46 / 259)$, faecal $(22.6 \%, 19 / 84)$, sewage $(14.3 \%, 2 / 14)$ and tap water $(7.1 \%$, $1 / 14)$ were significantly $(p<0.003)$ resistant to at least three classes of antimicrobials. Resistance to tetracycline class were predominantly observed in faecal samples $(69 \%, 58 / 84)$, followed by cloacal $(64.1 \%, 166 / 259)$, sewage $(35.7 \%, 5 / 14)$, and tap water $(7.1 \%, 1 / 84)$, respectively. Sewage water $(\mathrm{OR}=7.22,95 \% \mathrm{CI}=0.95-151.21)$ had significant association with antimicrobial resistance (AMR) acquisition. Multivariate regression analysis identified that the risk factors including sewage samples $(\mathrm{OR}=7.43,95 \% \mathrm{CI}=0.96-156.87)$ and farm size are leading drivers of $E$. coli antimicrobial resistance in the participating states of east coast peninsular Malaysia. We observed that the resistance patterns of $E$. coli isolates against 12 panel antimicrobials are generally similar in all selected states of east coast peninsular Malaysia. The highest prevalence of resistance was recorded in tetracycline (91.2\%), oxytetracycline (89.1\%), sulfamethoxazole/trimethoprim (73.1\%), doxycycline (63\%), and sulfamethoxazole $(63 \%)$. A close association between different risk factors and the high prevalence of antimicrobial-resistant $E$. coli strains reflects increased exposure to resistant bacteria and suggests a concern over rising misuse of veterinary antimicrobials that may contribute to the future threat of emergence of multidrug-resistant pathogen isolates. Public health interventions to limit antimicrobial resistance need to be tailored to local poultry farm practices that affect bacterial transmission.
\end{abstract}

Keywords: antimicrobial resistant; Escherichia coli; distribution; poultry farms; environment; east coast of peninsular Malaysia 


\section{Introduction}

Antimicrobial resistance (AMR) is of concern to global health security [1]. The persistence and emergence of antimicrobial resistance in bacterial communities with special reference to faecal form indicators pose a threat to treatment options of microbial infections, and thus place a burden on health services in human and animal health settings [2]. Moreover, the production of poultry for food relies on the use of antimicrobials to ensure animal health and growth promotion under intensive farming conditions [3]. Most of these antimicrobial compounds used in poultry operations are accumulated and biomagnified through the food chain. Exposure among local human populations to low levels of antimicrobial environmental contaminants through marine and agricultural ecosystems has been proposed to lead to development and acquisition of resistant bacteria [4-6].

Escherichia coli (E. coli) is an important pollution indicator with pathogenic strains responsible for food poisoning and food related infections. In upper middle-income countries, E. coli is responsible for $25 \%$ of infant diarrhea and some enteropathogenic, enter invasive, and enterotoxigenic types of $E$. coli are leading causes of food-borne diarrhea [7]. The prevalence of AMR among food-borne pathogens has increased during recent decades [7,8]. Factors influencing bacterial resistance on farms are substantial, including flock health status, farm management practices, and the environment [9]. Practices such as rampant use of broad-spectrum antimicrobials administered in low doses for growth promotion [10-13] and use of non-approved drugs or drugs used in off-label scenarios are driving the emergence of antimicrobial resistance in veterinary settings [14]. The tangled interplay of antimicrobial use and microbial transmission between people, animals, and the environment complicates efforts to reduce the development of AMR.

In Malaysia, these issues are likely to be most acute in poultry operations. Use of antimicrobials is frequently coupled with a high prevalence of infectious disease [15]. Malaysia has one of the largest poultry industries in South East Asia. Rapid growth and intensification in the production of chickens for food has the potential to increase the risk of development of AMR strains of pathogenic bacteria. In particular, the east coast of Malaysia has experienced rapid technological, genetic, management, and structural changes within the poultry production industry. In the absence of coordinated and systematically implemented regulation, AMR has been consistently reported at high levels from the poultry industry. However, little is known about the risk factors of AMR in poultry operations in peninsular Malaysia. Comprehensive examinations of a range of ultimate and proximate drivers of AMR at the poultry farm level have not been thoroughly investigated in South East Asia. To better understand the interactions of factors driving AMR across smallholders and poultry operations in Malaysia, we aimed to identify risk factors associated with the carriage of resistant $E$. coli isolates to help inform antimicrobial stewardship policy in poultry farms in Malaysia.

\section{Results}

We administered a semi-structured questionnaire to 31 poultry farmers and sampled only conveniently 14 farms with a total of 371 samples across three states of peninsular Malaysia. The socio-demographic traits of poultry farmers participating in the surveys are given in Table S1. Of these 371 samples from 14 poultry farms, the following types were collected: cloacal swabs $=259$; faecal $=84$; Sewage $=14$, tap water $=14$. A total of 717 E. coli samples were isolated from poultry and environmental samples, as follows: $(72 \%, 519 / 717)$ in cloacal swab; $(24 \%, 172 / 717)$ in faecal; $20(2.8 \%, 20 / 717)$ in tap water; and $6(0.83 \%, 6 / 717)$ in sewage system. A summary of the prevalence of tested $E$. coli samples were given in Table 1. The prevalence of E. coli among Kelantanese farms $(72.8 \%, 115 / 158)$ was higher than those of Terengganu (57.5\%), 46/80) and Pahang (57.9\%, 77/113) (Table 1). Among the districts, the highest prevalence of $E$. coli was recorded in Jeli farms $(88.5 \%, 23 / 26)$ followed by Machang $(85.7 \%, 24 / 28)$ and Kuala Terengganu $(76.9 \%, 20 / 26)$, respectively (Table 1$)$. Similarly, the prevalence of $E$. coli was higher in cloacal $(66.4 \%, 259 / 172)$ and faecal samples $(69 \%, 58 / 84))$ than sewage $(35.7 \%, 5 / 14)$ and tap water $(21.4 \%, 3 / 14)$ (Table 1$)$. A high 
prevalence of resistance to common antimicrobials was observed with special reference to tetracycline $(91.4 \%)$, oxytetracycline $(88.4 \%)$, sulfamethoxazole/trimethoprim $(74.2 \%)$, doxycycline $(66.4 \%)$, and sulfamethoxazole $(65.5 \%)$, ampicillin $(51.9 \%)$, and nalidixic acid $(52.2 \%)$, but there is a low resistance to chloramphenicol $(26.3 \%)$, gentamicin $(23.3 \%)$, amoxicillin $(21.2 \%)$, ciprofloxacin $(19.4 \%)$, and cefoxitin (6.5\%) (Figure 1). We observed that the resistance patterns of $E$. coli isolates against 12 panel antimicrobials are generally similar in all selected states of east coast peninsular Malaysia that include Kelantan, Terengganu, and Pahang. However, the prevalence of resistance to tetracycline, oxytetracycline, sulfamethoxazole/Trimethoprim, sulfamethoxazole, and doxycycline was consistently higher than other tested antimicrobials across selected states of east coast peninsular Malaysia (Figure 2). The percentage of antimicrobial-resistant $E$. coli isolated from each sample are summarized in Figure 3. Cloacal and faecal samples had the highest percentage of resistance followed by sewage and tap water systems (Figure 3). The summary of resistance to at least one antimicrobial and their associated risk factors is shown in Table 2. We observed that the size of the farm $(p<0.023)$, and source of the water $(p<0.02)$, poultry origin $(p<0.01)$, and the source of the sample $(p<0.01)$ factors were significantly associated with at least one AMR (Table 2). Furthermore, our findings revealed that cloacal $(17.8 \%, 46 / 259)$, faecal $(22.6 \%$, $19 / 84)$, sewage $(14.3 \%, 2 / 14)$, and tap water $((7.1 \%, 1 / 14)$ were significantly $(p<0.003)$ associated with resistant to at least three classes of antimicrobial (Table 3). Resistance to tetracycline class were predominantly observed in faecal samples $(69 \%, 58 / 84)$, followed by cloacal $(64.1 \%, 166 / 259)$, sewage $(35.7 \%, 5 / 14)$, and tap water $(7.1 \%, 1 / 84)$, respectively (Table 3). Similarly, resistance to quinolones class was predominantly recorded in cloacal samples $(45.2 \%, 117 / 259)$, followed by faecal $(41.7 \%, 35 / 84)$, sewage $(7.1 \%, 1 / 14)$ and tap water $(7.1 \%, 1 / 14)$, respectively (Table 3$)$. Sewage water $(\mathrm{OR}=7.22,95 \% \mathrm{CI}=0.95-151.21)$ had an increased likelihood of AMR acquisition (Table 4).

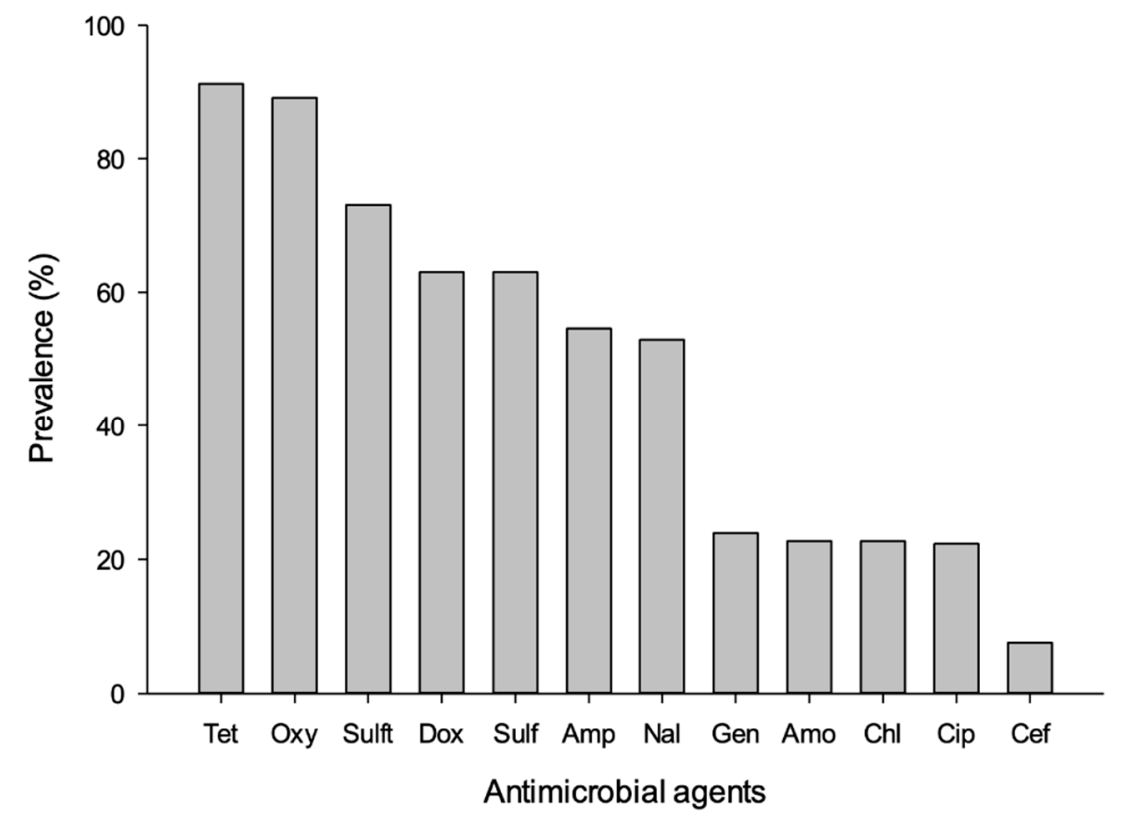

Figure 1. Prevalence of antimicrobial-resistant Escherichia coli isolated from poultry farms collected from Kelantan, Terengganu, and Pahang poultry operations. Data are the number of samples $(n=371)$. Tet: Tetracycline; Oxy: Oxytetracycline; Sulft: Sulfamethoxazole/trimethoprim; Sul: Sulfamethoxazole; Dox: Doxycycline; Amp: Ampicillin; Nal: Nalidixic acid; Chl: chloramphenicol; Gen: Gentamycin; Cip: Ciprofloxacin; Amo: amoxicillin and Cef: cefoxitin. 
Table 1. Summary of risk factors of E.coli among poultry farms in the Kelantan, Terengganu and Pahang, Malaysia $(n=371)$ by using chi-square test.

\begin{tabular}{|c|c|c|c|}
\hline Risk Factors & Samples Tested & Affected (\%) & $p$-Value \\
\hline Age & & & 0.511 \\
\hline Young & 187 & $123(65.8 \%)$ & \\
\hline Adult & 184 & $115(62.5 \%)$ & \\
\hline Management system & & & 0.541 \\
\hline Intensive & 187 & $115(61.5 \%)$ & \\
\hline Semi-intensive & 158 & $105(66.5 \%)$ & \\
\hline Mixed & 26 & $18(69.2 \%)$ & \\
\hline Production system & & & 0.278 \\
\hline Broiler & 212 & $129(60.8 \%)$ & \\
\hline Layer & 53 & $35(66 \%)$ & \\
\hline Mixed & 106 & $74(69.8 \%)$ & \\
\hline State & & & 0.012 \\
\hline Kelantan & 158 & $115(72.8 \%)$ & \\
\hline Terengganu & 80 & $46(57.5 \%)$ & \\
\hline Pahang & 133 & $77(57.9 \%)$ & \\
\hline Districts & & & 0.001 \\
\hline Bachok & 52 & $36(69.2 \%)$ & \\
\hline Kota Bharu & 26 & $18(69.2 \%)$ & \\
\hline Machang & 28 & $24(85.7 \%)$ & \\
\hline Pasir Mas & 26 & $14(53.8 \%)$ & \\
\hline Jeli & 26 & $23(88.5 \%)$ & \\
\hline Kuantan & 79 & $49(62 \%)$ & \\
\hline Pekan & 54 & $28(51.9 \%)$ & \\
\hline kuala terengganu & 26 & $20(76.9 \%)$ & \\
\hline Marang & 54 & $26(48.1 \%)$ & \\
\hline Sample source & & & 0.001 \\
\hline Cloaca swab & 259 & $172(66.4 \%)$ & \\
\hline Faecal Sample & 84 & $58(69 \%)$ & \\
\hline Sewage & 14 & $5(35.7 \%)$ & \\
\hline Tape Water & 14 & $3(21.4 \%)$ & \\
\hline Farm size & & & 0.013 \\
\hline Small & 104 & $77(74 \%)$ & \\
\hline Medium & 188 & $119(63.2 \%)$ & \\
\hline Large & 79 & $42(53.2 \%)$ & \\
\hline Origin of the poultry & & & 0.005 \\
\hline Local & 26 & $18(69.2 \%)$ & \\
\hline Imported & 133 & $71(53.4 \%)$ & \\
\hline Both & 212 & $149(70.3 \%)$ & \\
\hline
\end{tabular}




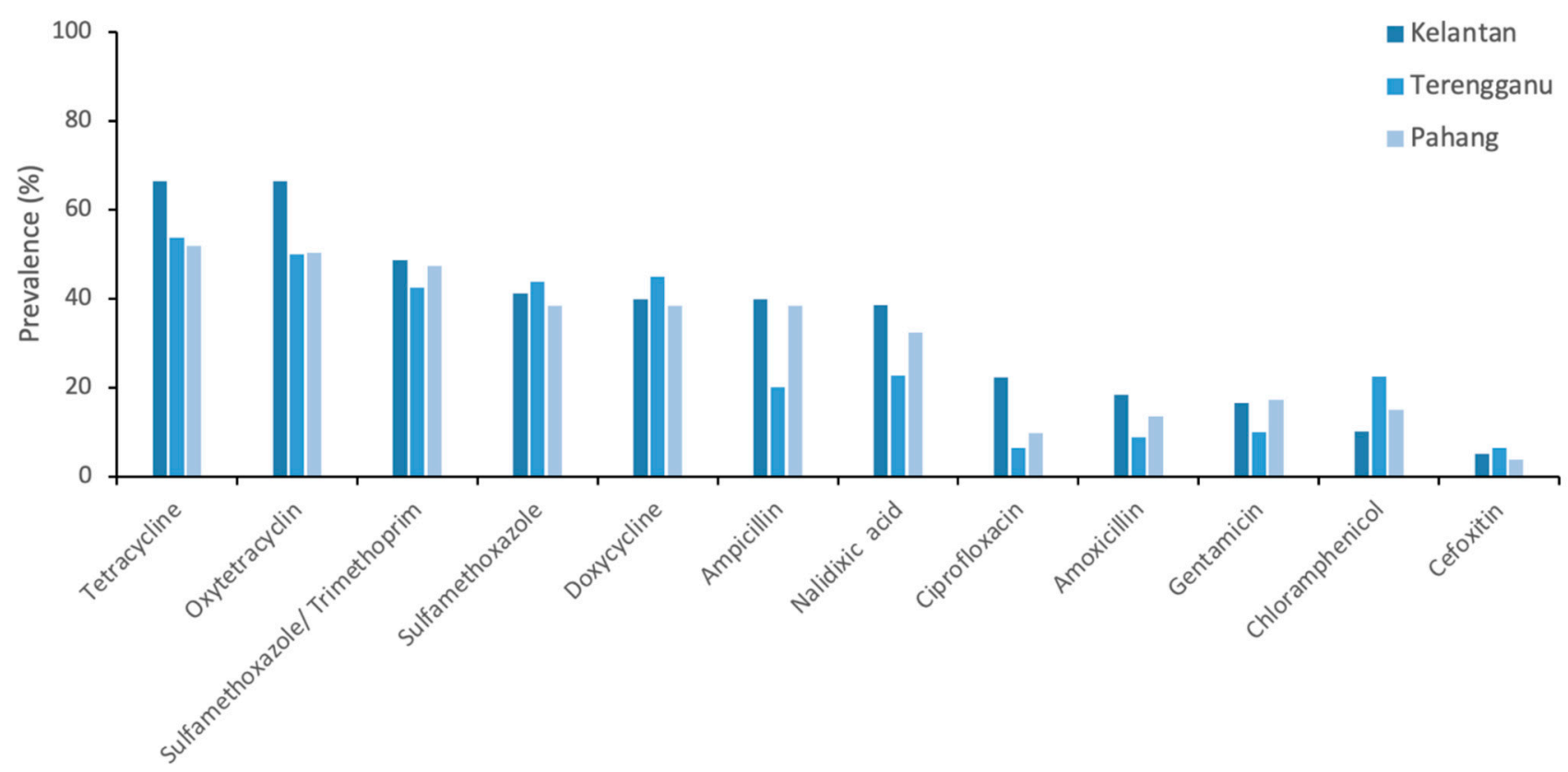

Figure 2. Prevalence of antimicrobial-resistant Escherichia coli isolated from poultry farms collected from Kelantan, Terengganu, and Pahang poultry operations. Data are the number of samples $(n=371)$.
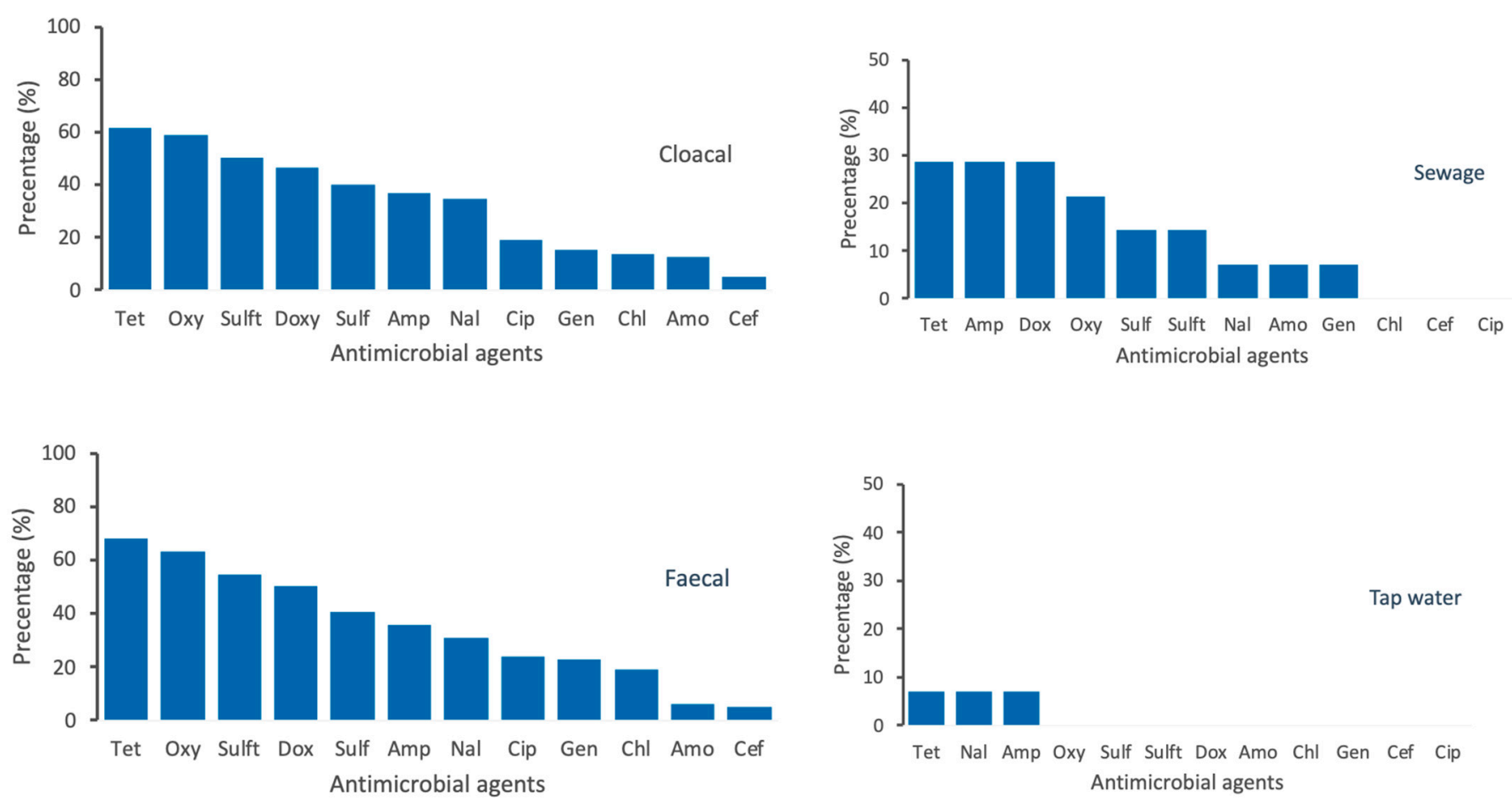

Figure 3. Percentage of antimicrobial-resistant Escherichia coli isolated from four epidemiological samples that include cloacal, faecal, tap water, and sewage collected from poultry farms. Data are the number of poultry samples $(n=371)$ in three states of east coast peninsular Malaysia. Tet: Tetracycline; Oxy: Oxytetracycline; Sulft: Sulfamethoxazole/trimethoprim; Sul: Sulfamethoxazole; Dox: Doxycycline; Amp: Ampicillin; Nal: Nalidixic acid; Chl: chloramphenicol; Gen: Gentamycin; Cip: Ciprofloxacin; Amo: amoxicillin and Cef: cefoxitin. 
Table 2. Summary of prevalence of resistance to at least one antimicrobial and their associated risk factors.

\begin{tabular}{|c|c|c|c|}
\hline Risk Factors & $\begin{array}{c}\text { No Antimicrobial } \\
\text { Resistance } \\
\mathbf{n}=137\end{array}$ & $\begin{array}{c}\text { Resistance to at least } \\
\text { One Antimicrobial } \\
n=234\end{array}$ & $p$-Value \\
\hline Age & & & 0.44 \\
\hline Young & $65(47.4 \%)$ & $122(52.1 \%)$ & \\
\hline Adult & $72(52.6 \%)$ & $112(47.9 \%)$ & \\
\hline Origin of the poultry & & & 0.01 \\
\hline Local & $10(7.3 \%)$ & $16(6.8 \%)$ & \\
\hline Imported & $63(46 \%)$ & $70(29.9 \%)$ & \\
\hline Both & $64(46.7 \%)$ & $148(63.2 \%)$ & \\
\hline Management system & & & 0.18 \\
\hline Intensive & $80(58.4 \%)$ & $115(49.1 \%)$ & \\
\hline Semi-intensive & $47(34.3 \%)$ & $103(44 \%)$ & \\
\hline Mixed & $10(7.3 \%)$ & $16(6.8 \%)$ & \\
\hline Production system & & & 0.21 \\
\hline Broiler & $86(62.8 \%)$ & $125(53.4 \%)$ & \\
\hline Layer & $18(13.1 \%)$ & $37(15.8 \%)$ & \\
\hline Mixed & $33(24.1 \%)$ & $72(30.8 \%)$ & \\
\hline Farm size & & & 0.02 \\
\hline Small & $29(21.2 \%)$ & $75(32.1 \%)$ & \\
\hline Medium & $70(51.1 \%)$ & $117(50 \%)$ & \\
\hline Large & $38(27.7 \%)$ & $42(17.9 \%)$ & \\
\hline Source of sample & & & $<0.001$ \\
\hline Cloacal swab & $89(65 \%)$ & $170(72.6 \%)$ & \\
\hline Faecal sample & $26(19 \%)$ & $58(24.8 \%)$ & \\
\hline sewage & $9(6.6 \%)$ & $5(2.1 \%)$ & \\
\hline Tap water & $13(9.5 \%)$ & $1(0.4 \%)$ & \\
\hline Water source & & & 0.02 \\
\hline Surface water & $37(27 \%)$ & $69(29.5 \%)$ & \\
\hline Bond water & $61(44.5 \%)$ & $72(30.8 \%)$ & \\
\hline Pump water & $39(28.5 \%)$ & $93(39.7 \%)$ & \\
\hline Sewage system & & & 0.60 \\
\hline Excellent & $38(27.7 \%)$ & $71(30.3 \%)$ & \\
\hline Good & $82(59.9 \%)$ & $128(54.7 \%)$ & \\
\hline Poor & $17(12.4 \%)$ & $35(15 \%)$ & \\
\hline Feed source & & & 0.53 \\
\hline Endogenous & $50(36.5 \%)$ & $82(35 \%)$ & \\
\hline Exogenous & $75(54.7 \%)$ & $138(59 \%)$ & \\
\hline Other & $12(8.8 \%)$ & $14(6 \%)$ & \\
\hline
\end{tabular}


Table 3. Summary of univariate analysis of poultry samples for antimicrobial-resistant $E$. coli from poultry farms in east coast of Malaysia ( $\mathrm{n}=371$ samples).

\begin{tabular}{|c|c|c|c|c|c|}
\hline Antimicrobials & $\begin{array}{l}\text { Cloacal } \\
n=259\end{array}$ & $\begin{array}{l}\text { Faecal } \\
\mathrm{n}=84\end{array}$ & $\begin{array}{c}\text { Sewage } \\
\mathrm{n}=14\end{array}$ & $\begin{array}{c}\text { Tape Water } \\
\mathbf{n}=\mathbf{1 4}\end{array}$ & $p$-Value \\
\hline No identified resistance & & & & & $<0.001$ \\
\hline No antimicrobial resistance & $89(34.4 \%)$ & $26(31 \%)$ & $9(64.3 \%)$ & $13(92.9 \%)$ & \\
\hline $\begin{array}{c}\text { Resistance to at least one } \\
\text { antimicrobial }\end{array}$ & $170(65.6 \%)$ & $58(69 \%)$ & $5(35.7 \%)$ & $1(7.1 \%)$ & \\
\hline Antimicrobial class resistance & & & & & 0.003 \\
\hline No antimicrobial resistance & $89(34.4 \%)$ & $26(31 \%)$ & $9(64.3 \%)$ & $13(92.9 \%)$ & \\
\hline Resistant to 1 class & $4(1.5 \%)$ & $1(1.2 \%)$ & $0(0 \%)$ & $0(0 \%)$ & \\
\hline Resistant to 2 classes & $13(5 \%)$ & $3(3.6 \%)$ & $2(14.3 \%)$ & $0(0 \%)$ & \\
\hline Resistant to 3 classes & $46(17.8 \%)$ & $19(22.6 \%)$ & $2(14.3 \%)$ & $1(7.1 \%)$ & \\
\hline Resistant to 4 classes & $74(28.6 \%)$ & $19(22.6 \%)$ & $1(7.1 \%)$ & $0(0 \%)$ & \\
\hline Resistant to 5 or more classes & $33(12.7 \%)$ & $16(19 \%)$ & $0(0 \%)$ & $0(0 \%)$ & \\
\hline Source of antimicrobials & & & & & 1 \\
\hline Drug supplier & $112(43.2 \%)$ & $36(42.9 \%)$ & $6(42.9 \%)$ & $6(42.9 \%)$ & \\
\hline Feed store & $147(56.8 \%)$ & $48(57.1 \%)$ & $8(57.1 \%)$ & $8(57.1 \%)$ & \\
\hline Tetracyclines & & & & & $<0.001$ \\
\hline Not resistant & $93(35.9 \%)$ & $26(31 \%)$ & $9(64.3 \%)$ & $13(92.9 \%)$ & \\
\hline Resistant & $166(64.1 \%)$ & $58(69 \%)$ & $5(35.7 \%)$ & $1(7.1 \%)$ & \\
\hline Penicillins & & & & & 0.048 \\
\hline Not resistant & $151(58.3 \%)$ & $47(56 \%)$ & $10(71.4 \%)$ & $13(92.9 \%)$ & \\
\hline Resistant & $108(41.7 \%)$ & $37(44 \%)$ & $4(28.6 \%)$ & $1(7.1 \%)$ & \\
\hline Aminoglycosides & & & & & 0.246 \\
\hline Not resistant & $219(84.6 \%)$ & $68(81 \%)$ & $13(92.9 \%)$ & $14(100 \%)$ & \\
\hline Resistant & $40(15.4 \%)$ & $16(19 \%)$ & $1(7.1 \%)$ & $0(0 \%)$ & \\
\hline Quinolones & & & & & 0.002 \\
\hline Not resistant & $142(54.8 \%)$ & $49(58.3 \%)$ & $13(92.9 \%)$ & $13(92.9 \%)$ & \\
\hline Resistant & $117(45.2 \%)$ & $35(41.7 \%)$ & $1(7.1 \%)$ & $1(7.1 \%)$ & \\
\hline Sulfonamides & & & & & $<0.001$ \\
\hline Not resistant & $104(40.2 \%)$ & $29(34.5 \%)$ & $11(78.6 \%)$ & $14(100 \%)$ & \\
\hline Resistant & $155(59.8 \%)$ & $55(65.5 \%)$ & $3(21.4 \%)$ & $0(0 \%)$ & \\
\hline Cephelosporins & & & & & 0.645 \\
\hline Not resistant & $246(95 \%)$ & $79(94 \%)$ & $14(100 \%)$ & $14(100 \%)$ & \\
\hline Resistant & $13(5 \%)$ & $5(6 \%)$ & $0(0 \%)$ & $0(0 \%)$ & \\
\hline Other classes & & & & & 0.025 \\
\hline Not resistant & $224(86.5 \%)$ & $65(77.4 \%)$ & $14(100 \%)$ & $14(100 \%)$ & \\
\hline Resistant & $35(13.5 \%)$ & $19(22.6 \%)$ & $0(0 \%)$ & $0(0 \%)$ & \\
\hline
\end{tabular}

Bacteria in samples obtained from young chickens (OR $=1.2,95 \% \mathrm{CI}=0.79-1.84)$ had an increased likelihood of AMR compared to samples from adult chickens (Table 4). Of note, in unadjusted analysis, there was no important difference in the odds of sampled E. coli having identified in AMR between intensive, mixed OR $=1.11,95 \% \mathrm{CI}=0.49-2.66$, or semi-intensive farms. Similarly, no difference in unadjusted analysis was observed in the production system. The results of the multivariate regression analysis adjusting for the size of the farm identified that the risk factors include the source of samples with special reference sewage samples $(\mathrm{OR}=7.43,95 \% \mathrm{CI}=0.96-156.87)$ and farm size (small $=\mathrm{OR}=$ $2.50,95 \% \mathrm{CI}=1.33-4.77$; medium $=\mathrm{OR}=1.55,95 \% \mathrm{CI}=0.89-2.67$ ) as leading drivers of E. coli antimicrobial resistance in the participating states of east coast peninsular Malaysia (Table 5).

For PCR analysis, the resistance genes, aac (3)- $I V$ for gentamicin, tet $(A)$ and tet $(B)$ for tetracyclines, catA1 for chloramphenicol, and sul1 for sulfonamides were investigated and the proportion of positive resistance genes were given in Table 6. Our results revealed 100\% 
positive amplicons for the sul1 gene, followed by aac (3)-IV 87\%, $64.2 \%$ of the E. coli isolates carried tet $(A)$ and tet $(B)$ (Table 6). The set of primers used for each gene is given in Table 7.

Table 4. Univariate regression analysis of risk factors for antimicrobial-resistant E. coli from poultry farms in east coast of Malaysia ( $\mathrm{n}=238$ samples).

\begin{tabular}{|c|c|c|c|c|c|}
\hline Variables & OR & $2.5 \%$ & $97.5 \%$ & $\operatorname{Pr}(>|z|)$ & \\
\hline \multicolumn{6}{|l|}{ Farms } \\
\hline Farm 1 & 10.20 & 2.95 & 42.89 & $<0.001$ & $* * *$ \\
\hline Farm 2 & 2.72 & 0.91 & 8.53 & 0.07 & ${ }^{\circ}$ \\
\hline Farm 3 & 13.03 & 3.46 & 65.56 & $<0.001$ & $* * *$ \\
\hline Farm 4 & 1.98 & 0.66 & 6.09 & 0.22 & \\
\hline Farm 5 & 2.31 & 0.78 & 7.18 & 0.134 & \\
\hline Farm 6 & 7.14 & 2.16 & 27.16 & 0.002 & $* *$ \\
\hline Farm 7 & 2.89 & 0.97 & 9.02 & 0.05 & . \\
\hline Farm 8 & Ref & Ref & Ref & Ref & \\
\hline Farm 9 & 4.61 & 1.48 & 15.63 & 0.01 & * \\
\hline Farm 10 & 3.82 & 1.25 & 12.52 & 0.02 & $*$ \\
\hline Farm 11 & 1.16 & 0.38 & 3.53 & 0.78 & \\
\hline Farm 12 & 1.06 & 0.34 & 3.25 & 0.91 & \\
\hline Farm 13 & 5.66 & 1.78 & 20.13 & 0.004 & $* *$ \\
\hline Farm 14 & 2.26 & 0.77 & 6.87 & 0.13 & \\
\hline \multicolumn{6}{|l|}{ Sample source } \\
\hline Cloaca swab & 24.83 & 4.82 & 454.74 & 0.002 & $* *$ \\
\hline Faecal sample & 29.0 & 5.35 & 540.73 & 0.001 & $* *$ \\
\hline Sewage & 7.22 & 0.95 & 151.21 & 0.09 & . \\
\hline Tap water & Ref & Ref & Ref & Ref & \\
\hline \multicolumn{6}{|l|}{ Age } \\
\hline Young & 1.21 & 0.79 & 1.84 & 0.38 & \\
\hline Adult & Ref & Ref & Ref & Ref & \\
\hline \multicolumn{6}{|l|}{ Poultry origin } \\
\hline Local & 1.44 & 0.61 & 3.50 & 0.41 & \\
\hline Both & 2.08 & 1.32 & 3.26 & 0.001 & $* *$ \\
\hline Imported & Ref & Ref & Ref & Ref & \\
\hline \multicolumn{6}{|c|}{ Management system } \\
\hline Semi-intensive & 1.52 & 0.97 & 2.39 & 0.06 & . \\
\hline Mixed & 1.11 & 0.48 & 2.65 & 0.80 & \\
\hline Intensive & Ref & Ref & Ref & Ref & \\
\hline \multicolumn{6}{|l|}{ Production system } \\
\hline Layer & 1.41 & 0.76 & 2.69 & 0.27 & \\
\hline Broiler & Ref & Ref & Ref & 0.11 & \\
\hline Mixed & 1.50 & 0.91 & 2.48 & Ref & \\
\hline \multicolumn{6}{|l|}{ Farm size } \\
\hline Small & 2.33 & 1.27 & 4.35 & 0.001 & $* *$ \\
\hline Medium & 1.51 & 0.88 & 2.57 & 0.125 & \\
\hline Large & Ref & Ref & Ref & Ref & \\
\hline \multicolumn{6}{|l|}{ Water source } \\
\hline Surface water & 1.57 & 0.93 & 2.68 & 0.08 & . \\
\hline Pump water & 2.02 & 1.22 & 3.36 & 0.01 & $* *$ \\
\hline \multicolumn{6}{|l|}{ Bond water } \\
\hline \multicolumn{6}{|l|}{ Sewage system } \\
\hline Excellent & 0.91 & 0.44 & 1.81 & 0.786 & \\
\hline Good & 0.75 & 0.39 & 1.42 & 0.398 & \\
\hline Poor & Ref & Ref & Ref & Ref & \\
\hline
\end{tabular}

Signif. codes: $0^{\star * * * \prime} 0.001^{\star * * \prime} 0.01^{\star * \prime} 0.05^{\prime \prime} .^{\prime} 0.1^{\prime \prime} 1$. 
Table 5. Multivariate regression analysis of risk factors for antimicrobial-resistant E. coli from poultry farms in east coast of Malaysia.

\begin{tabular}{|c|c|c|c|c|c|}
\hline & OR & $2.5 \%$ & $97.5 \%$ & $\operatorname{Pr}(>|z|)$ & \\
\hline Cloaca swab & 26.50 & 5.08 & 487.69 & 0.001 & ** \\
\hline Feacal sample & 30.92 & 5.63 & 579.63 & 0.001 & ** \\
\hline Sewage & 7.43 & 0.96 & 156.87 & 0.09 & . \\
\hline Farm size Small & 2.50 & 1.33 & 4.77 & 0.004 & ** \\
\hline Farm size medium & 1.55 & 0.89 & 2.67 & 0.114 & \\
\hline
\end{tabular}

Table 6. Comparison for the detection of resistance genes from samples using PCR.

\begin{tabular}{cccc}
\hline Antimicrobial Class/Agent & Resistance Gene & \% Isolates & Total \# Tested \\
\hline Gentamicin & aac(3)-IV & $12(85.7 \%)$ & 14 \\
Tetracyclines & tet(A), tet(B) & $9(64.2 \%)$ & 14 \\
Chloramphenicol & catA1 & $2(14.2 \%)$ & 14 \\
Sulfonamides & sul1 & $14(100 \%)$ & 14 \\
$\beta$-Lactams & blaSHV & $6(42.8 \%)$ & 14 \\
Trimethoprim & dhfrI & $4(28.5 \%)$ & 14 \\
\hline
\end{tabular}

Table 7. The set of primers used for each gene.

\begin{tabular}{|c|c|c|c|c|}
\hline Genes & Primer Sequence $\left(5^{\prime}\right.$ to $\left.3^{\prime}\right)$ & PCR Condition & Product Size & References \\
\hline$\beta$-Lactams & $\begin{array}{l}\text { F- CTATCGCCAGCAGGATCTGG } \\
\text { R- ATTTGCTGATTTCGCTCGGC }\end{array}$ & $\begin{array}{l}3 \text { min at } 95^{\circ} \mathrm{C} ; 35 \text { cycles of } 1 \\
\text { min at } 94{ }^{\circ} \mathrm{C}, 90 \mathrm{~s} \text { at } 55^{\circ} \mathrm{C} \text { and } \\
1 \text { min at } 72^{\circ} \mathrm{C} ; 10 \text { min at } 72{ }^{\circ} \mathrm{C}\end{array}$ & 543 & [16] \\
\hline $\begin{array}{l}\text { Gentamicin } \\
\operatorname{aac}(3)-I V\end{array}$ & $\begin{array}{l}\text { F-CTTCAGGATGGCAAGTTGGT } \\
\text { R-TCATCTCGTTCTCCGCTCAT }\end{array}$ & $\begin{array}{l}3 \text { min at } 95^{\circ} \mathrm{C} ; 35 \text { cycles of } 1 \\
\text { min at } 94^{\circ} \mathrm{C}, 90 \mathrm{~s} \text { at } 55^{\circ} \mathrm{C} \text { and } \\
1 \mathrm{~min} \text { at } 72^{\circ} \mathrm{C} ; 10 \mathrm{~min} \text { at } 72^{\circ} \mathrm{C}\end{array}$ & 286 & [17] \\
\hline $\begin{array}{l}\text { Sulfonamide } \\
\text { sul1 }\end{array}$ & $\begin{array}{l}\text { F- ACTGCAGGCTGGTGGTTATG } \\
\text { R- ACCGAGACCAATAGCGGAAG }\end{array}$ & $\begin{array}{c}3 \text { min at } 95 \mathrm{C} ; 35 \text { cycles of } 1 \\
\text { min at } 94 \mathrm{C}, 90 \mathrm{~s} \text { at } 55^{\circ} \mathrm{C} \text { and } 1 \\
\text { min at } 72{ }^{\circ} \mathrm{C} ; 10 \text { min at } 72{ }^{\circ} \mathrm{C}\end{array}$ & 271 & [8] \\
\hline $\begin{array}{l}\text { Tetracycline } \\
\quad \operatorname{tet}(A)\end{array}$ & $\begin{array}{l}\text { F-CCTCAATTTCCTGACGGGCT } \\
\text { R-GGCAGAGCAGGGAAAGGAAT }\end{array}$ & $\begin{array}{l}3 \text { min at } 95^{\circ} \mathrm{C} ; 35 \text { cycles of } 1 \\
\text { min at } 94 \mathrm{C}, 90 \mathrm{~s} \text { at } 55{ }^{\circ} \mathrm{C} \text { and } 1 \\
\min \text { at } 72{ }^{\circ} \mathrm{C} ; 10 \text { min at } 72{ }^{\circ} \mathrm{C}\end{array}$ & 712 & [18] \\
\hline $\begin{array}{l}\text { Tetracycline } \\
\text { tet }(B)\end{array}$ & $\begin{array}{l}\text { F-ACCACCTCAGCTTCTCAACG } \\
\text { R-GTAAAGCGATCCCACCACCA }\end{array}$ & $\begin{array}{l}3 \text { min at } 95^{\circ} \mathrm{C} ; 35 \text { cycles of } 1 \\
\text { min at } 94 \mathrm{C}, 90 \mathrm{~s} \text { at } 55^{\circ} \mathrm{C} \text { and } 1 \\
\min \text { at } 72^{\circ} \mathrm{C} ; 10 \text { min at } 72^{\circ} \mathrm{C}\end{array}$ & 586 & [18] \\
\hline $\begin{array}{l}\text { Chloramphenicol } \\
\quad \text { cat } A 1\end{array}$ & $\begin{array}{l}\text { F- GAAAGACGGTGAGCTGGTGA } \\
\text { R- TAGCACCAGGCGTTTAAGGG }\end{array}$ & $\begin{array}{l}3 \text { min at } 95^{\circ} \mathrm{C} ; 35 \text { cycles of } 1 \\
\text { min at } 94^{\circ} \mathrm{C}, 90 \mathrm{~s} \text { at } 55^{\circ} \mathrm{C} \text { and } \\
1 \mathrm{~min} \text { at } 72^{\circ} \mathrm{C} ; 10 \text { min at } 72^{\circ} \mathrm{C}\end{array}$ & 473 & [8] \\
\hline $\begin{array}{l}\text { Trimethoprim } \\
\text { dhfrI }\end{array}$ & $\begin{array}{l}\text { F-AAGAATGGAGTTATCGGGAATG } \\
\text { R-GGGTAAAAACTGGCCTAAAATTG }\end{array}$ & $\begin{array}{l}15 \mathrm{~min} \text { at } 95^{\circ} \mathrm{C} ; 30 \text { cycles of } 30 \\
\mathrm{~s} \text { at } 94^{\circ} \mathrm{C} ; 30 \mathrm{~s} \text { at } 58^{\circ} \mathrm{C} ; 1 \mathrm{~min} \\
\text { at } 72^{\circ} \mathrm{C} ; 10 \mathrm{~min} 72^{\circ} \mathrm{C} \text {. }\end{array}$ & 391 & [8] \\
\hline $\begin{array}{l}\text { Ampicillin } \\
\text { CITM }\end{array}$ & $\begin{array}{c}\text { F-TGGCCAGAACTGACAGGCAAA } \\
\text { R-TTTCTCCTGAACGTGGCTGGC }\end{array}$ & $\begin{array}{c}15 \mathrm{~min} \text { at } 95^{\circ} \mathrm{C} ; 30 \text { cycles of } 30 \\
\text { s at } 94{ }^{\circ} \mathrm{C} ; 30 \mathrm{~s} \text { at } 58{ }^{\circ} \mathrm{C} ; 1 \mathrm{~min} \\
\text { at } 72^{\circ} \mathrm{C} ; 10 \mathrm{~min} 72^{\circ} \mathrm{C} \text {. }\end{array}$ & 462 & [8] \\
\hline E.coli & $\begin{array}{l}\text { F-TGACGTTACCCGCAGAAGAA } \\
\text { R- CTCCAATCCGGACTACGACG }\end{array}$ & $\begin{array}{c}3 \mathrm{~min} \text { at } 95^{\circ} \mathrm{C} ; 35 \text { cycles of } 15 \mathrm{~s} \\
\text { at } 95^{\circ} \mathrm{C}, 90 \mathrm{~s} \text { at } 55^{\circ} \mathrm{C} \text { and } 15 \mathrm{~s} \\
\text { at } 72^{\circ} \mathrm{C} ; 10 \text { min at } 72^{\circ} \mathrm{C}\end{array}$ & 832 & [19] \\
\hline O157 & $\begin{array}{l}\text { F-GTGTCCATTTATACGGACATCCATG } \\
\text { R-CCTATAACGTCATGCCAATATTGCC }\end{array}$ & $\begin{array}{l}2 \mathrm{~min} \text { at } 94{ }^{\circ} \mathrm{C} ; 35 \text { cycles of } 30 \mathrm{~s} \\
\text { at } 94{ }^{\circ} \mathrm{C}, 30 \mathrm{~s} \text { at } 55^{\circ} \mathrm{C} \text { and } 30 \mathrm{~s} \\
\text { at } 72{ }^{\circ} \mathrm{C} ; 5 \text { min at } 72{ }^{\circ} \mathrm{C}\end{array}$ & 292 & [20] \\
\hline
\end{tabular}




\section{Discussion}

In this study, we observed that the resistance patterns of E. coli isolates against 12 panel antimicrobials are generally similar in all selected states of east coast peninsular Malaysia that include Kelantan, Terengganu, and Pahang. However, there is substantial heterogeneity in the prevalence of E. coli AMR within and between these states. These differences in prevalence across these states are linked to geographic-specific risk factors. The prevalence of E. coli resistance to tetracycline, oxytetracycline, sulfamethoxazole/trimethoprim, doxycycline, and sulfamethoxazole was highly consistent in all three participating states. This resistance also reflects the common use of antimicrobials in these poultry operations as well as in other agricultural activities [21]. Moreover, most of these antimicrobials are also used in human medicine with special reference to tetracycline, oxytetracycline, sulfamethoxazole, and ampicillin. Our findings are similar to those of other studies in poultry farms in low-income settings in South East Asia (SEA) [22]. For instance, poultry sampling farms in Vietnam found similar proportions of E. coli resistant to ampicillin (86.0\%), tetracycline $(93.4 \%)$ oxytetracycline $(93.6 \%)$, trimethoprim/sulfamethoxazole $(69.7 \%)$, nalidixic acid $(80.1 \%)$, gentamicin $(19.9 \%)$, and chloramphenicol (51.5\%). These farm-level estimates are based on non-randomly selected samples and we should expect these estimates to be higher than estimates from random collected datasets [22,23]. For example, E. coli isolated from poultry specimens presented at a veterinary clinic in the northern region of peninsular Malaysia were highly resistant to ampicillin (92.7\%), tetracycline (91.6\%), doxycycline (86.4), and gentamicin (41.6) [24]. Implementation of biosecurity levels including sewage system, visitors, PPE, washing facilities, use of disinfectant, and source of the food were not important factors of E. coli antimicrobial resistant in the sampled poultry farms. Furthermore, the prevalence of $E$. coli resistance in cloacal, faecal, sewage, and tap water isolates were significantly $(p<0.003)$ associated with AMR acquisition. Importantly, sewage isolates (OR $=7.43,95 \% \mathrm{CI}=0.96-156.8)$ had an increased testing of AMR as the sewage systems nearby these farms were identified as important risk factors for the presence of AMR. The resistance data from sewage samples can be augmented well with data from clinical based surveillance [25]. The lower prevalence in sewage and tap water isolates, however, could be correlated with sensitivity as it is likely lower than isolate-based surveillance [26]. Resistance to tetracycline class were predominantly observed in faecal isolates, followed by cloacal, sewage, and tap water, respectively. Similarly, resistance to quinolones class were predominantly recorded in cloacal isolates, followed by faecal, sewage, and tap water, respectively.

The source of water and the presence of a sewage system were identified as important risk factors for the presence of AMR in E. coli isolates in the study sites. For instance, the pump water $\mathrm{OR}=2.02,95 \% \mathrm{CI}=1.22-3.36, p<0.000$ and surface water $\mathrm{OR}=1.57,95 \%$ $\mathrm{CI}=0.93-2.68, p<0.000$ was significantly associated with AMR acquisition. Furthermore, we detected residual amounts of the antimicrobials tested in the water systems of these premises and alongside the tributaries in the nearby rivers, which is in close proximity to livestock operations (data not presented here). Most of these antimicrobial residues belong to Sulfonamides and Quinolones in the surface water at an average concentration range of 5 to $85 \mathrm{ng} \mathrm{L}^{-1}$. Conversely, we have detected low levels of Tetracyclines in the surface water, although higher levels of TCs were detected in the faecal samples of poultry operations and in the Kelantanese tributaries sediments. The discovery of these antimicrobial contents could be most likely attributed to potential contaminations from livestock farming discharge field runoff. Importantly, the sampled poultry farms usually access drinking water from intact sources, and thus the association could reflect contact transmission at the farm level. This association has important implications for lowincome countries, where potable water remains a pressing challenge [27]. Consumption of poultry meat and its products is increasing, and most poultry meat and eggs are produced and distributed through informal sources that operate outside national quality-control standards and regulations [28]. 
Nonetheless, it is worth noting that our study is comparable within the local context of East coast peninsular Malaysia but that there are limited studies conducted in these areas $[29,30]$. Pathogen transmission could lead to rampant use of veterinary antimicrobials by these farmers as our self-reported data did show explicitly such association. In our findings, we have observed the association between antimicrobial-resistant E. coli and the type of production system, although such phenomena were not consistent across all tested antimicrobials. Importantly, although there was a link between washing facilities and antimicrobial-resistant bacteria, such an association, however, was not an important factor for pathogen transmission dynamics. Interestingly, small scale poultry farms in the selected states were far more likely to carry AMR-resistant E. coli $(\mathrm{OR}=2.33,95 \% \mathrm{CI}=1.27-4.35)$ than medium and large scales farms. The poultry farms practicing intensive management system and the samples obtained from young chickens had increased odds of testing positive for antimicrobial resistant E. coli. Our findings highlight that the current strategies to tackle global antimicrobial resistance should include identifying the persistence and drivers of antimicrobial resistance within the context of cultural and management practices in the relevant communities.

Use of antimicrobials was very high in our survey (100\%) and $64.5 \%$ of participants reported that they had received them from a regulated drug supplier (Table S1). This suggests that many small holders may buy unregulated medicine from black markets and thus contributes to the development of AMR. Furthermore, it also reflects the national need for a policy to regulate the safety of antimicrobials and guidance for usage and sale. A "One Health" approach involving different actors such as human and veterinary medicine, agriculture, finance, environment, and consumers will be a utopian model to combat global AMR.

In the current study, the sul1 gene was detected in $100 \%$ using the conventional PCR from the poultry samples of east coast peninsular Malaysia. Similarly, the aac (3)$I V$ was detected in $87 \%$ where $64.2 \%$ of the $E$. coli isolates carried tet $(A)$ and tet $(B)$. However, it is worth noting that there are no comparable existing studies which have investigated the presence of these genes in poultry operations of east coast peninsular Malaysia. The detection of resistant E. coli genes in rural surface water which is in close proximity to poultry operations remains a source of concern and suggests a potential pool of veterinary antimicrobials and resistant bacteria to the community. This study demonstrated the rampant use of veterinary antimicrobials in poultry operations, which is probably responsible for AMR in community settings. A close association between different risk factors and the high prevalence of antimicrobial-resistant $E$. coli strains reflects the increased exposure to resistant bacteria and suggests a concern over rising misuse of veterinary antimicrobials that may result in a future threat of emergence of multidrugresistant pathogen isolates. Public health interventions to limit antimicrobial resistance need to be tailored to local poultry farm practices that affect bacterial transmission.

\section{Materials and Methods}

\subsection{Study Area}

The study was carried out between 8 February 2019 and 23 February 2020 in poultry farms located in three states of East coast peninsular Malaysia: Kelantan, Terengganu, and Pahang (Figure 4). These three states border the South China Sea and are dominated by a tropical climate which is characterized by humidity. There is a heavy monsoon season from November to March every year. The average temperature ranges from 21 to $32{ }^{\circ} \mathrm{C}$. Average yearly rainfall falls is from $2032 \mathrm{~mm}$ to $2540 \mathrm{~mm}$, with the wettest months being from November through January. 


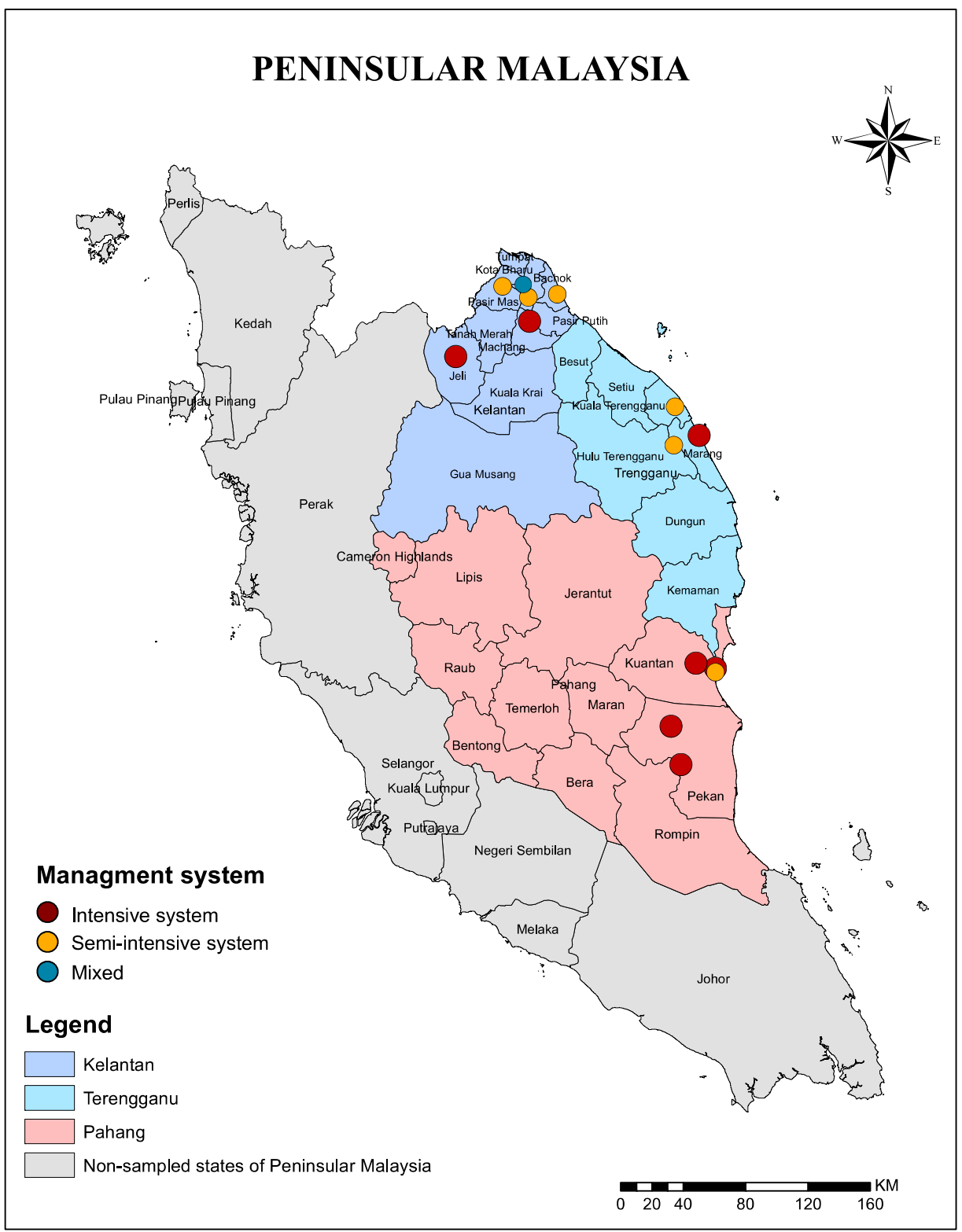

Figure 4. A map showing location of the sampled states and exact location 14 poultry farms sampled and their management systems in Kelantan, Terengganu, and Pahang of east cast peninsular Malaysia. The map was created using ArcGIS v. 10 (esri Inc., Redlands, CA, USA).

\subsection{Study Design, Definitions, and Data Sources}

We conducted a cross sectional survey targeting poultry farms in three states of east coast peninsular Malaysia that include Kelantan, Terengganu, and Pahang. A total of 371 samples (cloacal swabs $=259$; faecal $=84$; Sewage $=14$, tap water $=14$ ) were randomly collected. Farm characteristics including management, biosecurity, and disease history were collected using a semi-structured questionnaire. As such, 31 farmers that met strict inclusion criteria of keeping poultry farms and who responded to written consent were included in the analyses. Data pertaining to potential risk factors including management, biosecurity, and disease history were collected using semi-structured questionnaires. Similarly, antimicrobial usage data was obtained using a count-based approach, representing the use (yes/no) of an antimicrobial at the time of visit. Furthermore, sources from where antimicrobials and feed along with the source of water and the nature of their current sewage systems were collected (Table S1). 
Regarding the management system, flock size, and sewage system, the following definitions and criteria were used:

- Intensive management system is defined as mainly concentrated and often mechanized operations that use controlled-environment systems to provide the ideal thermal environment for the poultry.

- Semi-intensive system is that which relies on natural airflow though the shed for ventilation.

- Extensive system is mainly pasture-based and land-based where birds in the household flock are typically housed overnight in the shelter and are let out in the morning to forage during the day.

- The criteria of the farm size included large-scale commercial farms that has more than $\geq 10,000$ birds, medium-scale commercial farms that has more 5000-10,000, and small-scale farms where birds are often kept in single-age groups of $>1000$.

- A poor sewage system is defined as that which retains high volumes of wastewater with low flow rate, blackish appearance, and sewage smell odour as a result of composing agricultural waste-probably as leakage from nearby irrigated effluent which is used for agricultural land application along with the presence of food waste, green waste, plastic, and heavy materials.

- A good sewage system is that which has good drainage with no agricultural waste and relatively low heavy materials.

- Excellent swage system is that which has significant drainage, no agriculture, and heavy materials.

Briefly, the cloacal samples were collected using sterile transport media; faecal samples using sterile containers and water samples using sterile water bottles and kept in a cooling box containing ice bags maintaining low temperature at $\left(4^{\circ}\right)$ and transferred to the lab within $24 \mathrm{~h}$. All samples were collected according to standard operating procedures and good laboratory practices. A detailed study design is summarized in Figure 5.

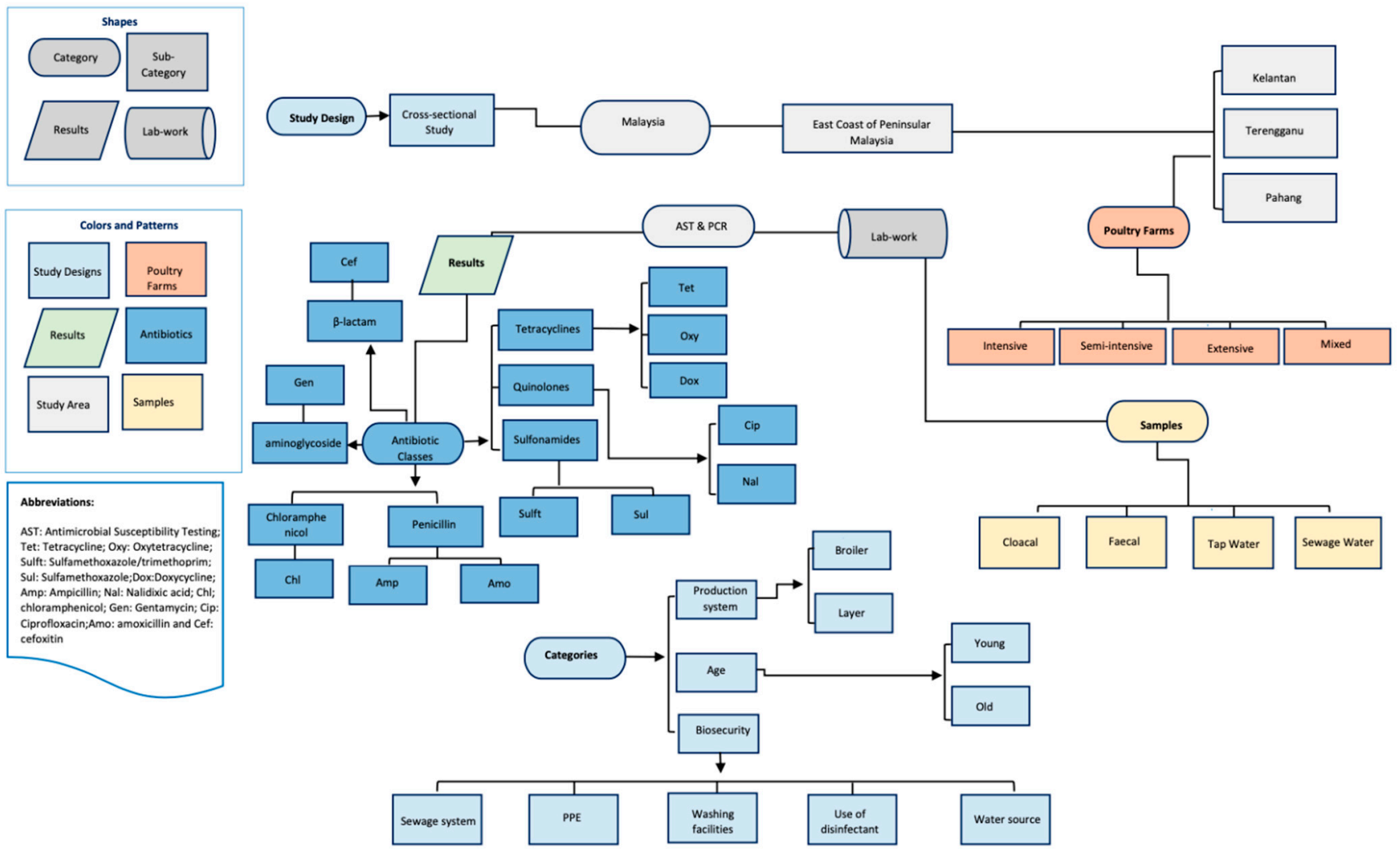

Figure 5. Diagrammatic representation of the study design. 


\subsection{Microbiological Testing}

All cloacal swabs and fresh faecal samples were placed in Amies transport media, and transported on ice to the molecular biology laboratory, Universiti Malaysia Kelantan (UMK). Sewage tap water and surface water samples were transported in conical tubes, all on ice. Samples were enriched in buffered peptone water for $24 \mathrm{~h}$ and then plated onto eosin methylene blue agar (EMBA) and incubated for $24 \mathrm{~h}$ at $37^{\circ} \mathrm{C}$. Subsequently, five colonies were selected and sub-cultured on EMBA, before being further sub-cultured on Müller-Hinton agar and stored at $-20{ }^{\circ} \mathrm{C}$ in cryovials. A single colony was picked at random from the plate for each original sample and biochemical tests including triple sugar iron agar, Simmon's citrate agar, and motility-indole-lysine media were used for presumptive identification of E. coli isolates. All isolates were revived and inoculated onto Müller-Hinton plates before antimicrobial susceptibility testing.

\subsection{Antimicrobial Susceptibility Testing}

Isolates were tested for susceptibility against a panel of 12 antimicrobial agents perceived to be used frequently in both human and veterinary medicine in Malaysia. These antimicrobials included ampicillin $(10 \mu \mathrm{g})$, amoxicillin-clavulanic acid $(20 / 10 \mu \mathrm{g})$, chloramphenicol $(30 \mu \mathrm{g})$, gentamicin $(10 \mu \mathrm{g})$, tetracycline $(30 \mu \mathrm{g})$, Oxytetracycline $(30 \mu \mathrm{g})$, doxycycline $(30 \mu \mathrm{g})$, trimethoprim-sulfamethoxazole $(25 \mu \mathrm{g})$, nalidixic acid $(30 \mu \mathrm{g})$, ciprofloxacin $(5 \mu \mathrm{g})$, cefoxitin $(30 \mu \mathrm{g})$, and sulfonamides $(300 \mu \mathrm{g})$ using the disc diffusion method (DDM) according to the Clinical and Laboratory Standards Institute guidelines [31]. Clinical and Laboratory Standards Institute guidelines were also used to determine as breakpoints for classifying isolates as sensitive, intermediate, or resistant to the drug [31]. Multidrugresistant E. coli was defined as "non-susceptibility to at least one agent in three or more antimicrobial classes." An antibiogram was defined as the combination of antimicrobials to which an isolate was resistant, and thus antibiogram length was defined as the number of antimicrobials to which an isolate was phenotypically resistance.

\subsection{PCR}

\subsubsection{DNA Extraction of Escherichia Coli Isolates}

E. coli isolates were sub-cultured overnight in Luria-Bertani broth and genomic DNA was extracted using a Presto ${ }^{\mathrm{TM}}$ Mini gDNA Bacteria Kit according to the manufacturer's instructions.

\subsubsection{Primers and PCR Assay for Specific Genes}

The incidence of genes related to resistance to gentamicin (aac (3)-IV), tetracyclines (tet (A) and tet (B)), chloramphenicol (catA1 and cmlA), and sulfonamides (sul1) was determined by PCR. The set of primers used for each gene is shown in Table 6. PCR reactions were performed in a total volume of $25 \mathrm{~mL}$ using GoTaq1 Green Master Mix (Promega, USA), including $12.5 \mathrm{~mL}$ of GoTaq1 Green Master Mix, $1 \mathrm{~mL}$ of forward primer, $1 \mathrm{~mL}$ of reverse primers, $5.5 \mathrm{~mL}$ of nuclease-free water, and $5 \mathrm{~mL}$ of extracted DNA. Amplification reactions were carried out using a DNA thermocycler (Fisher Scientific UK, Loughborough, UK). PCR amplification was performed in duplicate. Amplified samples were analysed by electrophoresis in $1.5 \%$ agarose gel and were stained with ethidium bromide.

\subsection{Statistical Analysis}

Data were entered into Microsoft Excel spreadsheet and imported into SPSS version 25 and the R software (version 3.6.1) for statistical analysis. The data were sorted and checked for consistency and duplication. Data visualization were done in ArcGIS v. 10 (esri Inc., Redlands, CA, USA). The data focused on sets of variables that have been previously proposed or identified as risk factors for antimicrobial resistance [32,33]. Briefly, we have classified resistance as no resistance to antimicrobials detected in isolates and categorized the antimicrobials into their classes then identified which isolates were resistant to one or more specific classes. Classes of antimicrobials included tetracyclines, penicillins, aminogly- 
cosides, quinolones, sulfonamides, third generation cephalosporins, and chloramphenicol. Prevalence of resistance of E.coli to a panel of 12 antimicrobials was also compared between four epidemiological samples that include cloaca, faecal, tap water, and sewage samples. Descriptive statistics for frequency of association between AMR and potential risk factors was performed. Selection of variables for inclusion in a logistic regression model were based on prior hypotheses and variables which were suggestive of an important effect from the descriptive analysis.

Supplementary Materials: The following are available online at https:/ /www.mdpi.com/2079-638 2/10/2/117/s1, Table S1: Characteristics of 31 farmers in Kelantan, Terengganu, and Pahang states, Malaysia.

Author Contributions: Conceptualization, A.Y.O. and I.O.; data curation, S.A.E. and D.S.; formal analysis, S.A.E., D.S. and A.Y.O.; funding acquisition, A.Y.O. and I.O.; investigation, S.A.E.; methodology, S.A.E. and D.S.; project administration, A.Y.O.; resources, I.O.; supervision, M.A.K., R.K., A.Y.O. and I.O.; validation, D.S. and A.Y.O.; visualization, A.Y.O.; writing-original draft, A.Y.O.; writing-review and editing, L.E., N.H., M.M.A.H., Y.A.S. and A.Y.O. All authors have read and agreed to the published version of the manuscript.

Funding: This research received research fund from Research Management Centre, Universiti Malaysia Kelantan (R/SGJP/A06.00/01625A/001/2018/000459/). David Simons, Linzy Elton, Najmul Haider, Muzamil Mahdi Abdel Hamid, Richard Kock and Abdinasir Yusuf Osman are members of the Pan African Network for Rapid Research, Response, and Preparedness for Infectious Diseases Epidemics consortium (PANDORA-ID-NET) — supported by the European and Developing Countries Clinical Trials Partnership (EDCTP2) programme (RIA2016E-1609. The funders had no role in the study design, data collection, analysis, interpretation or writing of the manuscript.

Institutional Review Board Statement: “This study was approved by Institutional Research Ethics Committee of the Faculty of Veterinary Medicine, University Malaysia Kelantan (UMK) (Ref: 12/2018).

Informed Consent Statement: Informed consent was obtained from all subjects involved in the study.

Data Availability Statement: The data for the present study will be available upon reasonable request.

Acknowledgments: David Simons, Linzy Elton, Najmul Haider, Muzamil Mahdi Abdel Hamid, Richard Kock and Abdinasir Yusuf Osman are members of the Pan African Network for Rapid Research, Response, and Preparedness for Infectious Diseases Epidemics consortium (PANDORA-IDNET) - supported by the European and Developing Countries Clinical Trials Partnership (EDCTP2) programme (RIA2016E-1609). We acknowledge the LC-MS/MS Laboratory, Monash University, Malaysia and the support of the Faculty of Veterinary Medicine, Universiti Malaysia Kelantan with special reference to the field work assistance offered by Muhammad Luqman Nordin and Mohd Farhan Hanif. Abdinasir Yusuf Osman is partially supported by the IsDB, Saudi Arabia and the Centre for Global Public Health, Institute of Population Health Science, Queen Mary, University of London, UK.

Conflicts of Interest: The authors declare that they have no competing interests.

\section{References}

1. Prestinaci, F.; Pezzotti, P.; Pantosti, A. Antimicrobial resistance: A global multifaceted phenomenon. Pathog. Glob. Health 2015, 109, 309-318. [CrossRef] [PubMed]

2. Holmes, A.H.; Moore, L.S.; Sundsfjord, A.; Steinbakk, M.; Regmi, S.; Karkey, A.; Guerin, P.J.; Piddock, L.J. Understanding the mechanisms and drivers of antimicrobial resistance. Lancet 2016, 387, 176-187. [CrossRef]

3. Winckler, C.; Grafe, A. Use of veterinary drugs in intensive animal production. J. Soils Sediments 2001, 1, 66. [CrossRef]

4. Sun, P.; Barmaz, D.; Cabrera, M.L.; Pavlostathis, S.G.; Huang, C.-H. Detection and quantification of ionophore antibiotics in runoff, soil and poultry litter. J. Chromatogr. A 2013, 1312, 10-17. [CrossRef] [PubMed]

5. Kay, P.; Blackwell, P.A.; Boxall, A.B. Transport of veterinary antibiotics in overland flow following the application of slurry to arable land. Chemosphere 2005, 59, 951-959. [CrossRef] [PubMed] 
6. Phillips, I. Withdrawal of growth-promoting antibiotics in Europe and its effects in relation to human health. Int. J. Antimicrob. Agents 2007, 30, 101-107. [CrossRef]

7. Akbar, A.; Sitara, U.; Ali, I.; Khan, M.I.; Phadungchob, T.; Anal, A.K. Presence of Escherichia coli in poultry meat: A potential food safety threat. Int. Food Res. J. 2014, 21, 941.

8. Van, T.T.H.; Chin, J.; Chapman, T.; Tran, L.T.; Coloe, P.J. Safety of raw meat and shellfish in Vietnam: An analysis of Escherichia coli isolations for antibiotic resistance and virulence genes. Int. J. Food Microbiol. 2008, 124, 217-223. [CrossRef]

9. Acar, J.; Moulin, G. Antimicrobial resistance at farm level. Rev. Sci. Tech. 2006, 25, 775-792. [CrossRef]

10. Sarkar, P.; Gould, I.M. Antimicrobial agents are societal drugs. Drugs 2006, 66, 893-901. [CrossRef]

11. Hammerum, A.M.; Heuer, O.E.; Emborg, H.-D.; Bagger-Skjøt, L.; Jensen, V.F.; Rogues, A.-M.; Skov, R.L.; Agersø, Y.; Brandt, C.T.; Seyfarth, A.M. Danish integrated antimicrobial resistance monitoring and research program. Emerg. Infect. Dis. 2007, 13, 1633. [CrossRef] [PubMed]

12. Mathew, A.G.; Cissell, R.; Liamthong, S. Antibiotic resistance in bacteria associated with food animals: A United States perspective of livestock production. Foodborne Pathog. Dis. 2007, 4, 115-133. [CrossRef] [PubMed]

13. Prescott, J.F. Antimicrobial use in food and companion animals. Anim. Health Res. Rev. 2008, 9, 127. [CrossRef] [PubMed]

14. Donado-Godoy, P.; Gardner, I.; Byrne, B.A.; Leon, M.; Perez-Gutierrez, E.; Ovalle, M.; Tafur, M.; Miller, W. Prevalence, risk factors, and antimicrobial resistance profiles of Salmonella from commercial broiler farms in two important poultry-producing regions of Colombia. J. Food Prot. 2012, 75, 874-883. [CrossRef]

15. Planta, M.B. The role of poverty in antimicrobial resistance. J. Am. Board Fam. Med. 2007, 20, 533-539. [CrossRef]

16. Abo-Amer, A.E.; Shobrak, M.Y.; Altalhi, A.D. Isolation and antimicrobial resistance of Escherichia coli isolated from farm chickens in Taif, Saudi Arabia. J. Glob. Antimicrob. Resist. 2018, 15, 65-68. [CrossRef]

17. Messele, Y.E.; Abdi, R.D.; Yalew, S.T.; Tegegne, D.T.; Emeru, B.A.; Werid, G.M. Molecular determination of antimicrobial resistance in Escherichia coli isolated from raw meat in Addis Ababa and Bishoftu, Ethiopia. Ann. Clin. Microbiol. Antimicrob. 2017, 16, 55. [CrossRef]

18. Torkan, S.; Khamesipour, F.; Anyanwu, M. Detection of virulence and antibacterial resistance genes in Salmonella isolates from diarrhoeic dogs in Iran. Rev. Méd. Vét. 2015, 166, 221-228.

19. Aliyu, A.; Saleha, A.; Jalila, A.; Zunita, Z. Risk factors and spatial distribution of extended spectrum $\beta$-lactamase-producingEscherichia coli at retail poultry meat markets in Malaysia: A cross-sectional study. BMC Public Health 2016, 16, 699. [CrossRef]

20. Imtiaz, J.; Hashmi, I.; Saeed, A.; Qazi, I.; Arshad, M. Development of PCR Protocol for Detection of Escherichia Coli in Drinking Water. In Water Resources Management VII; Brebbia, C.A., Ed.; WIT PRESS: Southhampton, UK, 2013; pp. $225-232$.

21. Tang, K.L.; Caffrey, N.P.; Nóbrega, D.B.; Cork, S.C.; Ronksley, P.E.; Barkema, H.W.; Polachek, A.J.; Ganshorn, H.; Sharma, N.; Kellner, J.D. Restricting the use of antibiotics in food-producing animals and its associations with antibiotic resistance in food-producing animals and human beings: A systematic review and meta-analysis. Lancet Planet. Health 2017, 1, e316-e327. [CrossRef]

22. Usui, M.; Ozawa, S.; Onozato, H.; Kuge, R.; Obata, Y.; Uemae, T.; Ngoc, P.T.; Heriyanto, A.; Chalemchaikit, T.; Makita, K. Antimicrobial susceptibility of indicator bacteria isolated from chickens in Southeast Asian countries (Vietnam, Indonesia and Thailand). J. Vet. Med. Sci. 2014, 76. [CrossRef] [PubMed]

23. Nguyen, V.T.; Carrique-Mas, J.J.; Ngo, T.H.; Ho, H.M.; Ha, T.T.; Campbell, J.I.; Nguyen, T.N.; Hoang, N.N.; Pham, V.M.; Wagenaar, J.A. Prevalence and risk factors for carriage of antimicrobial-resistant Escherichia coli on household and small-scale chicken farms in the Mekong Delta of Vietnam. J. Antimicrob. Chemother. 2015, 70, 2144-2152. [PubMed]

24. Shahaza, O.; Zakirah, S.; Muhammad, A.; Syamsyul, A.; Maswati, M. Antimicrobial resistance in veterinary clinical isolates of Escherichia coli from northern region of Peninsular Malaysia. Malays. J. Vet. Res. 2017, 8, 1-7.

25. Cheong, Y.; Lim, V.; Jegathesan, M.; Suleiman, A. Antimicrobial resistance in 6 Malaysian general hospitals. Med. J. Malays. 1994, $49,317-326$.

26. Aarestrup, F.M.; Woolhouse, M.E. Using sewage for surveillance of antimicrobial resistance. Science 2020, 367, 630-632. [CrossRef] [PubMed]

27. Dangour, A.D.; Watson, L.; Cumming, O.; Boisson, S.; Che, Y.; Velleman, Y.; Cavill, S.; Allen, E.; Uauy, R. Interventions to improve water quality and supply, sanitation and hygiene practices, and their effects on the nutritional status of children. Cochrane Database Syst. Rev. 2013, 1. [CrossRef]

28. Chuah, L.-O.; He, X.B.; Effarizah, M.E.; Syahariza, Z.A.; Shamila-Syuhada, A.K.; Rusul, G. Mislabelling of beef and poultry products sold in Malaysia. Food Control 2016, 62, 157-164. [CrossRef]

29. Bong, C.W.; Chai, S.K.; Chai, L.C.; Wang, A.J.; Lee, C.W. Prevalence and characterization of Escherichia coli in the Kelantan River and its adjacent coastal waters. Water Supply 2020, 20, 930-942. [CrossRef]

30. Alhaj, N.; Mariana, N.; Raha, A.; Ishak, Z. Prevalence of antibiotic resistance among Escherichia coli from different sources in Malaysia. Int. J. Poult. Sci. 2007, 6, 293-297. [CrossRef]

31. Clinical and Laboratory Standards Institute. Performance Standards for Antimicrobial Susceptibility Testing, 26th ed; CLSI Supplement M100; CLSI: Wayne, PA, USA, 2016. 
32. Murphy, C.P.; Carson, C.; Smith, B.A.; Chapman, B.; Marrotte, J.; McCann, M.; Primeau, C.; Sharma, P.; Parmley, E.J. Factors potentially linked with the occurrence of antimicrobial resistance in selected bacteria from cattle, chickens and pigs: A scoping review of publications for use in modelling of antimicrobial resistance (IAM. AMR Project). Zoonoses Public Health 2018, 65, 957-971. [CrossRef]

33. Ibrahim, R.A.; Cryer, T.L.; Lafi, S.Q.; Basha, E.-A.; Good, L.; Tarazi, Y.H. Identification of Escherichia coli from broiler chickens in Jordan, their antimicrobial resistance, gene characterization and the associated risk factors. BMC Vet. Res. 2019, 15, 159. [CrossRef] [PubMed] 